\title{
Diversity of the parB and repA genes of the Burkholderia cepacia complex and their utility for rapid identification of Burkholderia
} cenocepacia

\author{
Pavel Drevinek ${ }^{1}$, Adam Baldwin ${ }^{2}$, Christopher G Dowson ${ }^{2}$ and \\ Eshwar Mahenthiralingam*1
}

\author{
Address: ${ }^{1}$ Cardiff School of Biosciences, Cardiff University, Cardiff, CF10 3TL, UK and ${ }^{2}$ Department of Biological Sciences, Warwick University, \\ Coventry, CV4 7AL, UK \\ Email: Pavel Drevinek - drevinekp@cardiff.ac.uk; Adam Baldwin - adam.baldwin@warwick.ac.uk; \\ Christopher G Dowson - c.g.dowson@warwick.ac.uk; Eshwar Mahenthiralingam* - MahenthiralingamE@cardiff.ac.uk \\ * Corresponding author
}

Published: 7 March 2008

BMC Microbiology 2008, 8:44 doi:10.1 I86/147I-2180-8-44

This article is available from: http://www.biomedcentral.com/I47|-2/80/8/44

(c) 2008 Drevinek et al; licensee BioMed Central Ltd.

This is an Open Access article distributed under the terms of the Creative Commons Attribution License (http://creativecommons.org/licenses/by/2.0), which permits unrestricted use, distribution, and reproduction in any medium, provided the original work is properly cited.

\begin{abstract}
Background: Burkholderia cenocepacia is the most prominent species of the B. cepacia complex $(\mathrm{Bcc})$, a group of nine closely related and difficult to identify bacteria that cause serious infections in patients with cystic fibrosis. Despite its clinical relevance, identification of $B$. cenocepacia as a single species is unavailable, as it splits by a widely used recA gene-based PCR identification method into discrete phylogenetic subgroups IIIA, IIIB, IIIC and IIID. With the aim of identifying gene targets suitable for unified detection of $B$. cenocepacia strains, we examined sequence polymorphisms in the repA and parB genes. These essential genes are involved in the replication and partitioning of bacterial replicons, hence we also had the opportunity for the first time to investigate the evolution of the multireplicon (three chromosome) structure of Bcc genomes.
\end{abstract}

Results: Alignment of the repA and parB genes from publicly available Bcc genome sequences enabled the design of primers for their amplification and sequence analysis. Multilocus sequencing typing, a highly discriminatory method for Bcc species and strain discrimination, was used to select strains of unique sequence types (STs) that spanned the known Bcc genetic diversity. Sequence datasets of repA ( 83 isolates, 67 STs) and parB ( 120 isolates, 95 STs) genes from the second chromosome were aligned and examined phylogenetically to identify polymorphisms suitable for identification of $B$. cenocepacia. In contrast to parB, the Bcc repA sequences demonstrated distinct clustering of $B$. cenocepacia from other species, which enabled the design a species-specific multiplex PCR. The novel single-reaction $B$. cenocepacia detection method was tested on a panel of I 42 different Bcc strains (I42 STs) and distinguished recA groups IIIA, IIIB and IIID, from all other Bcc members with $100 \%$ sensitivity and $93 \%$ specificity.

Conclusion: The repA-based multiplex PCR is a useful aid to the rapid identification of the most clinically relevant $B$. cenocepacia recA subgroups IIIA, IIIB and IIID. Phylogenetic analysis of repA and parB genes demonstrated that acquisition of the second and third replicons of Bcc genomes occurred prior to their differentiation into discrete species and that the sharing of replicons across species had not occurred. 


\section{Background}

Patients with cystic fibrosis (CF) are threatened over their lifetime with multiple respiratory infections contributing significantly to their morbidity and mortality [1]. One of the most problematic infectious agents are the microorganisms belonging to the Burkholderia cepacia complex (Bcc), a group of nine closely related bacterial species initially designated as genomovars prior to their formal naming [2]. Current species within the Bcc are: B. cepacia (genomovar I; [3]), B. multivorans (genomovar II; [3]), B. cenocepacia (genomovar III; [4]), B. stabilis (genomovar IV; [5]), B. vietnamiensis (genomovar $\mathrm{V} ;[3]$ ), B. dolosa (genomovar VI; [6]), B. ambifaria (genomovar VII; [7]), B. anthina (genomovar VIII; [8]) and B. pyrrocinia (genomovar IX; [8]). Although all Bcc species can be isolated from CF respiratory samples [2], two of them, B. cenocepacia and $B$. multivorans, are by far the most predominant, cumulatively accounting for approximately $85-98 \%$ of all Bcc infections in CF [9-12].

Within B. cenocepacia, phylogenetic analysis of the recA gene sequence [13] subdivides this species into four distinct clusters, IIIA, IIIB, IIIC and IIID [4]. A fifth B. cenocepacia subgroup, IIIE, has been described in multilocus sequence typing studies of the Bcc [14-16], however, the clustering of IIIE isolates in all these studies has been very distinct from B. cenocepacia subgroups IIIA to IIID. The classification of these isolates as $B$. cenocepacia IIIE is now recognised to have been a mis-assignment ( $P$. Vandamme, personal communication) and they will be referred as Bcc Group E until their taxonomic position is formally resolved. Epidemiological surveillance of $B$. cenocepacia $\mathrm{CF}$ infection has revealed the vast majority of cases to be caused by subgroups IIIA $[11,17,18]$ and IIIB $[10,12]$. Clinical strains of IIID origin were originally reported from a single CF centre [19] and to date no clinical IIIC strains have been described [20]. In addition to nine established Bcc species, other Bcc-related groups with unidentified genomovar status have been described such as Bcc Group K $[16,21]$ and Bcc groups 1 to $6[14,15]$; the phenotypic similarity of these novel groups with B. cenocepacia provides the potential for misidentification.

The need for accurate identification of Bcc has led to the development of several PCR-based methods $[13,22,23]$, aimed at preventing the alarming rate of Bcc misidentification based upon the use of standard phenotypic tests [24]. The house-keeping gene $r e c A$ whose polymorphisms allowed the discrimination of all the Bcc species [13] has been found to be particularly convenient for the Bcc identification purposes [2]. However, when the PCR targeting the recA is applied to detection of $B$. cenocepacia, it lacks the option to detect all isolates within this species in a single reaction. Separate PCR primers have to be employed for each existing recA variant from the B. cenocepacia IIIA and IIIB phylogenetic subgroups [13,21,25]; no PCR primers have been developed for IIIC or IIID strains. Multilocus sequence typing (MLST) analysis of seven housekeeping genes (including recA) [14] has now largely superseded recA gene analysis in terms of being a gold standard test for discriminatory species or novel group identification within the Bcc. MLST also has the great advantage of being capable of globally tracking and discriminating strains $[16,26]$. However, despite the fact that DNA sequence analysis is becoming more cost effective and accessible, the requirement for PCR and sequencing of seven genes is often beyond the means of standard diagnostic laboratories.

In this study we developed a PCR for simultaneous detection of all clinically relevant $B$. cenocepacia recA groups IIIA, IIIB and IIID [4]. We analyzed nucleotide polymorphisms of two additional essential genes, parB and repA, from the second chromosomal replicon of Bcc bacteria which are involved in the partitioning and replication, respectively, of their multipartite genomes [27]. Alignment of parB and repA genes from six sequenced Bcc genomes enabled the design of universal PCR primers capable of amplification of each respective gene from all Bcc bacteria. These PCRs were then used to generate Bcc sequence datasets for each gene. After subsequent detection and comparison of discriminatory nucleotide polymorphisms, the final design of a $B$. cenocepacia speciesspecific repA PCR was carried out. The availability of a collection of parB and repA sequences also allowed us to evaluate for the first time the genetic and evolutionary conservation of the second chromosome that is carried by all members of the Bcc $[2,27]$. The design of parB/repA PCRs, diagnostic evaluation of the performance of a $B$. cenocepacia-specific repA against a gold-standard set of isolates identified by MLST [14], and the evolutionary specificity of the second chromosome to each discrete Bcc species are described.

\section{Results}

Analysis of repA and parB genes encoded in Bcc genomes Six Bcc genomes that spanned B. cenocepacia, B. vietnamiensis, B. ambifaria and Bcc Group K were examined for the presence of repA and parB genes. Due to the multireplicon structure of Bcc genomes that are known to comprise at least 3 chromosomal replicons and may have one or more associated plasmids $[2,27]$, homologs of both genes were present in multiple copies. For all Bcc genomes examined, all replicons possessed their own sequence-specific copy of $p a r B$, and every replicon except the largest (chromosome 1), harboured a sequence-specific copy of repA. Phylogenetic analysis of the repA and parB sequences retrieved from the second and third chromosomal replicons was performed (Figure 1). Alleles for both genes clustered primarily by the replicon they were encoded on. Within these 


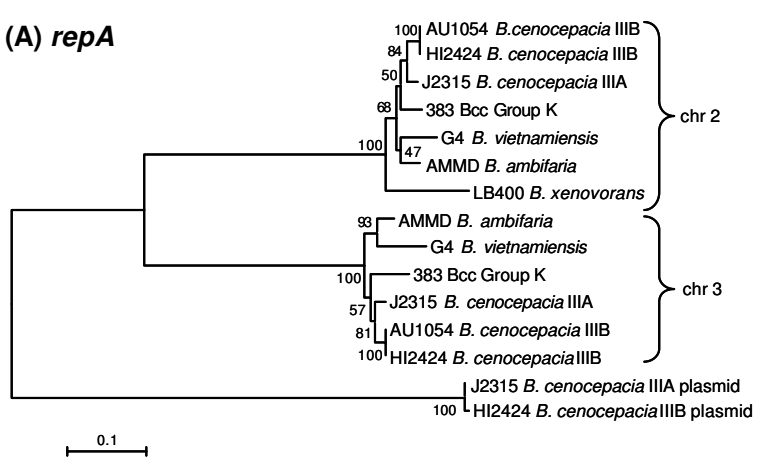

(B) parB

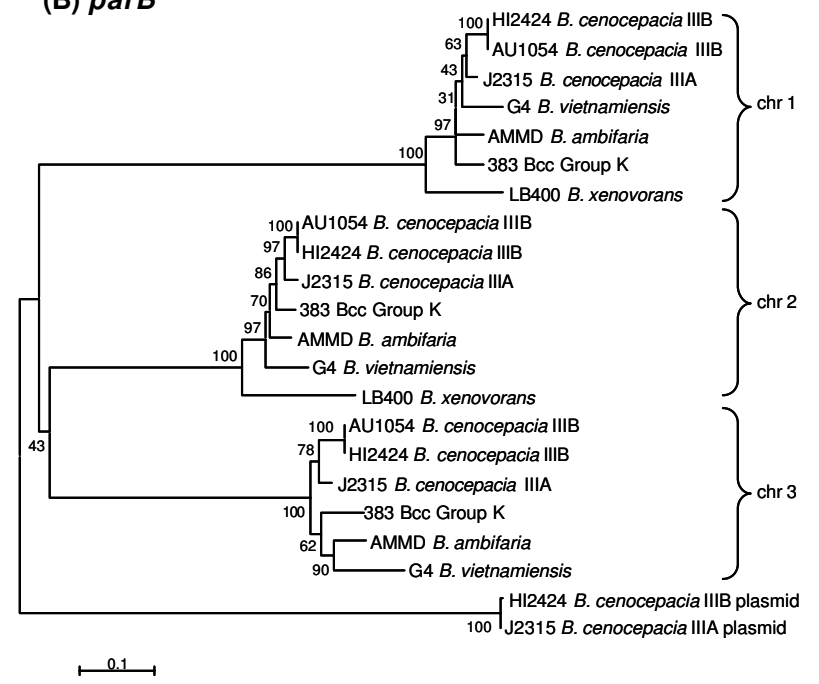

Figure I

Phylogenetic analysis of repA and parB genes obtained from completed Burkholderia genomes. The repA (panel A) and parB (panel B) from six B. cepacia complex genomes and $B$. xenovorans LB400 were analysed phylogenetically as described in the methods. The brackets indicate clustering of sequences by their replicon origin and the genetic distance scale is indicated.

replicon-specific clusters, each species was resolved as a discrete phylogenetic arm, with the three B. cenocepacia derived sequences clustering more closely than the Bcc Group K, B. vietnamiensis or B. ambifaria sequences. The repA and parB sequences on the respective plasmids within B. cenocepacia J2315 and HI2424 were also resolved and not as closely related as the chromosomally encoded genes.

The repA and parB genes present on chromosome 2 were selected for evaluation as targets to which both universal and B. cenocepacia specific PCRs could be designed. Using the alignment of full length Bcc genes, universal primers were designed to amplify $66 \%$ of the 1,368 bp repA coding sequence and $62 \%$ of the 1,062 bp parB sequence present on the second chromosome (Table 1); these primers were applied to collection of strains representative of the genetic diversity within the Bcc identified by MLST $[14,15,26]$. Sequence analysis of an internal 623 bp portion of $898 \mathrm{bp}$ repA product was performed using two further primers, while the shorter, 656 bp long parB amplicon was sequenced completely using the universal primers for this gene (Table 1).

\section{Sequence analysis of the Bcc repA gene}

The relationship and sequence similarities among repA alleles originating from different Bcc species was investigated for 67 Bcc ST types. Phylogenetic analyses of repA (Figure 2, panel A; raw sequences are provided in Additional file 1: Sequences repA) revealed that $B$. cenocepacia recA groups IIIA and IIIB clustered in close proximity to each other, indicating they might share unique sequence polymorphisms not present in other Bcc species/groups. The variation in repA alleles from $B$. cenocepacia IIIB was considerably greater than that seen in IIIA strains which were virtually identical for the 11 STs examined. B. cenocepacia IIIC and IIID, along with two Bcc5 strains of distinct ST clustered adjacent to IIIA and IIIB; a third Bcc5 ST formed the next most closely related arm of the tree (Figure 2, panel A). These five groups split weakly from all other Bcc species examined on the basis of the repA phylogeny.

The sequence length obtained from a subgroup of 73 isolates representing 60 ST types was suitable for MLST-like analysis. In an analogous fashion to the allele numbering system used in MLST [14], a unique number was assigned to every repA sequence variant and resulted in identification of 35 new repA alleles (submitted to Genbank as accession numbers EU165089 to EU165123). None of the repA alleles was able to further distinguish a single ST type and all isolates of the same ST always carried the same repA allele. Moreover, the same repA allele numbers were found shared by multiple STs within the same species. These data indicated the $\operatorname{rep} A$ gene was more conserved than the gltB, gyrB, lepA and $\operatorname{tr} p B$ genes used in conventional MLST of the Bcc.

\section{Phylogenetic analysis of parB gene}

The parB gene polymorphisms were examined in 95 different STs, including same isolates used for $r e p A$ analysis above. The constructed phylogenetic tree for parB (Figure 2, panel B; raw sequences are provided in Additional file 2: Sequences parB) demonstrated considerably weaker relatedness between $B$. cenocepacia IIIA and IIIB compared to the repA tree. IIIA and IIIB were not found next to each other and in addition, IIIB isolates were split into three separate parB clusters. B. cepacia, Bcc Group K, Bcc Group E, Bcc4, Bcc5 and Bcc6, were clustered between IIIA and 
Table I: Primers used in this study.

\begin{tabular}{|c|c|c|c|}
\hline Primer & Purpose & Sequence $\left(5^{\prime} \text { to } 3^{\prime}\right)^{a, b}$ & Product size (bp) \\
\hline repA-UNI-5 & universal repA PCR & GGA TGT GGT GAG TGC CAG TTC A & 898 \\
\hline repA-UNI-3 & universal repA PCR & CCG CTG YTC GGT CAT CTG C & \\
\hline repA-SEQ-5 & repA sequencing species-specific PCR & CAG CAG GCC GAC GAC TCG & 623 \\
\hline repA-SEQ-3 & repA sequencing & ATC GGC TGC TTG CGY TCG GT & \\
\hline repA-636-3 & species-specific PCR & GTC GAG CGC $\underline{G A G ~ C A T ~ C G G ~} \underline{C}$ & 636 \\
\hline repA-237-3 & species-specific PCR & CCA $\subseteq A C$ GCG GCG $\underline{\text { GGT }} \underline{\mathrm{GGT}}$ & 237 \\
\hline parB-UNI-5 & $\begin{array}{l}\text { universal parB PCR } \\
\text { parB sequencing }\end{array}$ & GCA GCA GGC GAT YCA C $\overline{\text { GTT G }}$ & 656 \\
\hline parB-UNI-3 & $\begin{array}{c}\text { universal parB PCR } \\
\text { parB sequencing }\end{array}$ & CGT CGC GCT TST CCT TCG G & \\
\hline
\end{tabular}

a Standard code, $\mathrm{Y}=\mathrm{C}$ or $\mathrm{T} ; \mathrm{S}=\mathrm{C}$ or $\mathrm{G}$

b Mis-matched nucleotide positions in the species specific primers are underlined

the three IIIB subgroups. This pronounced phylogenetic diversity showed that there was substantial sequence heterogeneity within the parB gene. Application of the MLSTlike allele designation system to the parB gene of 114 isolates (89 STs) resulted in the identification of 67 different alleles (submitted to Genbank as accession numbers EU165124 to EU165190). Similarly to the repA results, identical alleles were found present in more than one ST and the isolates of the same ST never differed by their parB allele. However, only one of the 7 MLST loci, gyrB, exceeded the number of different alleles found in parB.

\section{Design of PCR for B. cenocepacia IIIA, IIIB and IIID}

One of the aims of this study was to develop a single species-specific PCR for B. cenocepacia, primarily aimed at unifying the identification of the most clinically significant groups IIIA and IIIB that exist within this species. After obtaining the repA and $p a r B$ sequence datasets which spanned the current diversity within the Bcc, both genes were evaluated for their potential as PCR targets. However, the high degree of parB sequence heterogeneity within the IIIB group excluded this gene from being evaluated further as a suitable target.

In this respect, the repA proved to be less troublesome and allowed the design of $B$. cenocepacia-specific primers. However, even within repA, two sites with species-specific nucleotide polymorphisms had to be selected to avoid false positivity deriving from non-IIIA non-IIIB isolates. The first species-specific polymorphism site selected was targeted by primer repA-237-3 (Table 1); this primer had priming base mis-matches in the repA sequences of $B$. cepacia, B. multivorans, B. cenocepacia recA group IIIC, $B$. stabilis, B. vietnamiensis, B. dolosa, B. ambifaria, B. anthina, B. pyrrocinia, Bcc Group K, Bcc Group E and Bcc4. Primer repA-237-3 in combination with the sequencing forward primer repA-SEQ-5 was predicted to produce a $237 \mathrm{bp}$ product with IIIA, IIIB, and Bcc5 while the mis-matched species described above were predicted to be negative. The second site selected for the multiplex PCR was targeted by primer repA-636-3 (Table 1) and containing priming basis mis-matches present in the repA sequences of $B$. multivorans, B. cenocepacia IIIC and Bcc5; in combination with repA-SEQ-5, primer repA-636-3 was therefore predicted to produce a 636 bp product from all Bcc species except the latter three.

As a combined multiplex PCR, with repA-SEQ-5 as the forward primer and a mixture of repA-237-3/repA-636-3 as the reverse primers, the predicted identification algorithm from the in silico analysis was as follows: B. cenocepacia IIIA and IIIB isolates would produce both 636 and 237 bp PCR products; B. cenocepacia IIIC and B. multivorans would be negative for both; Bcc 5 would produce only the 237 bp product, while all the remaining Bcc species and novel groups would produce only the $636 \mathrm{bp}$ amplicon. At this stage, we could not predict outcomes for Bcc6 (due to a lack of sequence information) and B. cenocepacia IIID (the repA sequence obtained was sufficient for phylogenetic analysis but did not span the target regions selected). However, since we were mainly focused on the specific detection of B. cenocepacia IIIA and IIIB as predominant CF pathogens, the multiplex PCR was tested on a group of 83 isolates spanning the diversity of the Bcc (Figure 3). As predicted, B. cenocepacia recA groups IIIA and IIIB were characterized with two positive bands, and in addition subgroup IIID was also positive for both of these. B. cenocepacia IIIC and B. multivorans were negative for both PCR products and Bcc5 produced the $237 \mathrm{bp}$ product alone. All the remaining Bcc members were positive for just the $636 \mathrm{bp}$ amplicon (Figure 3).

\section{Specificity and sensitivity of B. cenocepacia PCR}

The initial success of the multiplex prompted a complete screen of a broad range of different STs representing all Bcc species and most of recently described Bcc groups which was used to determine specificity and sensitivity of the multiplex PCR. The hypothesis tested was that the novel repA PCR was capable of discriminating B. cenocepacia IIIA, IIIB and IIID from all other Bcc species and novel 

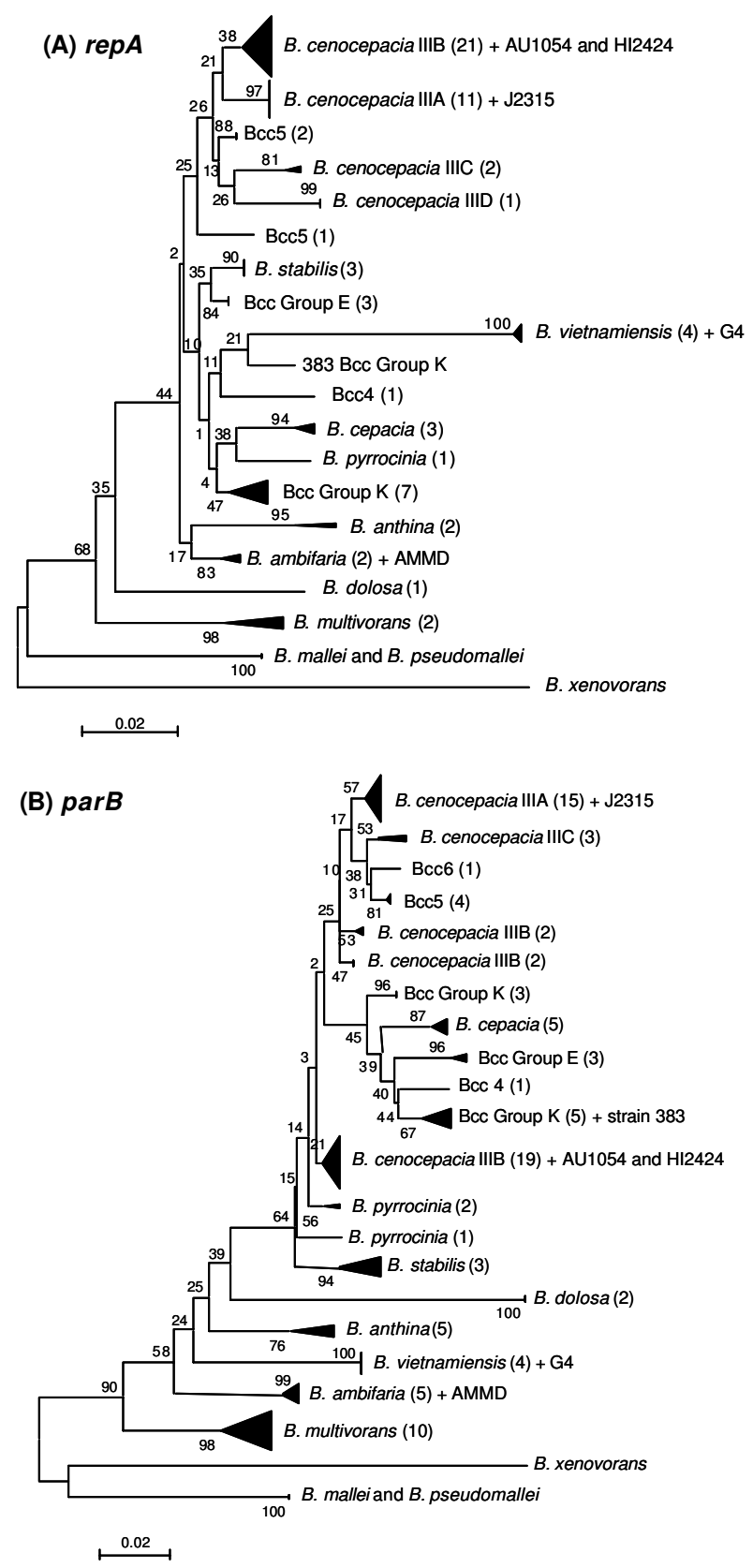

Figure 2

Diversity of $B$. cepacia complex repA and parB genes. Phylogenetic trees of repA (Panel A) and parB (panel B) genes determined from the $B c c$ isolates examined in this study were drawn as described in the methods. The number of STs examined for each distinct phylogenetic group is provided in brackets, followed by names of genomic reference strains where they were included in the analysis. The genetic distance scale is indicated. The B. mallei, B. pseudomallei and B. xenovorans repA or parB genes, respectively, were included as outgroups for each tree. groups (Table 2). For B. cenocepacia IIIA, IIIB and IIID, all 44 isolates (39 STs) gave the expected double-positive PCR result. All 3 B. cenocepacia IIIC isolates (3 STs) were negative. Outside of $B$. cenocepacia, 69 isolates (53 STs) from formally named species within the current $B$. cepacia complex produced results that correlated to their species designation (Figure 3). The only exceptions were 3 isolates representing $1 \mathrm{ST}$ from $B$. vietnamiensis, $B$. ambifaria and $B$. pyrrocinia, respectively, that were negative for both multiplex products (Table 2). Expansion of the test strain collection to novel Bcc groups with no formal species names demonstrated the presence of $B$. cenocepacia III-AB-D-like double positives only among 7 STs in group Bcc6. Three STs within the 12 Bcc5 isolates tested produced single 237 bp amplicons as expected, however, the remaining 6 STs were negative for both amplicons. The other novel groups screened all produced the expected multiplex PCR result.

In total, 135 out of 142 STs were assigned correctly into one of the two categories: (i) being $B$. cenocepacia IIIA, IIIB or IIID (39 out of 39 STs), and (ii) being other Bcc species or groups (96 out of 103 STs). This represented 100\% test sensitivity (39/39) and overall $93.2 \%$ specificity (96/ 103). The species-specific character of the assay was also further tested by examining amplification from closely related non-Burkholderia species and bacteria commonly encountered in CF sputum (see methods); all these microorganisms did not cross-react with novel multiplex PCR and designed primers. The detection threshold of the PCR which comprised just 20 cycles was found to be $10^{7} \mathrm{~B}$. cenocepacia CFU/mL; addition of 5 further PCR cycles reduced the minimal amount of bacteria needed for successful amplification to $10^{6} \mathrm{CFU} / \mathrm{mL}$.

\section{Discussion}

Correct identification of bacteria belonging to the B. cepacia complex still remains dependent on the use of molecular methods. The non-ribosomal house-keeping gene recA has become one of the mainstays of Bcc identification, since its sequence polymorphisms allow both detection of the whole complex and also species level identification $[13,21]$. However, one of the major drawbacks of using the recA gene was the fact that $B$. cenocepacia is split into four recA phylogenetic variants [4]. As a consequence, $B$. cenocepacia lacks a one-step identification test, even though this particular species represents the most frequent Bcc pathogen in persons with CF $[2,10-$ $12,17]$. A single test for unified identification of B. cenocepacia isolates, especially the clinically dominant subgroups IIIA and IIIB [10-12], would be a great asset to the rapid diagnosis of infection caused with these virulent $\mathrm{CF}$ pathogens. In this study we examined the nucleotide polymorphisms present in the essential house-keeping genes rep $A$ and $p a r B$, which are involved in the control of repli- 


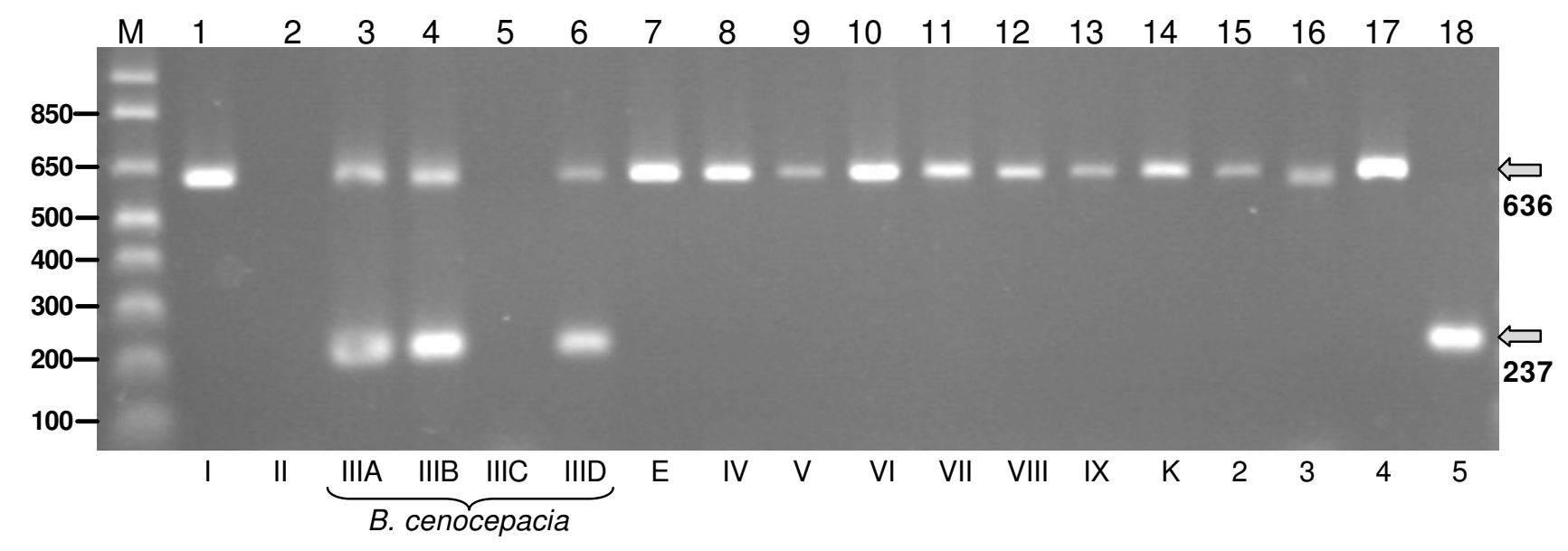

\section{Figure 3}

PCR products identifying $B$. cenocepacia produced by the multiplex repA PCR. Double band positivity was characteristic for B. cenocepacia IIIA, IIIB and IIID groups. All other species of Bcc and novel MLST-defined groups were either positive for one band or double negative (Table 2). Samples analysed in each lane are as follows (isolate number): $M$, I kb molecular size marker (relevant size fragments indicated in bP); I, B. cepacia (BCC0002); 2, B. multivorans (BCC08I4); 3, B. cenocepacia IIIA (BCCI 295); 4, B. cenocepacia IIIB (BCC0I87); 5, B. cenocepacia IIIC (BCC063 I); 6, B. cenocepacia IIID (BCC0506); 7, Bcc Group E (BCC0I 98); 8, B. stabilis (BCC0479); 9, B. vietnamiensis (BCC0I95); I0, B. dolosa (BCC0I6I); II, B. ambifaria (BCC0363); I 2, B. anthina (BCC0036); I3, B. pyrrocinia (BCC0488); I4, Bcc Group K (BCCI303); I5, BCC2 (BCC0484); I6, BCC3 (BCCI306); I7, BCC4 (BCC0405) and I8, BCC5 (BCC0397).

Table 2: Diversity of the Bcc collection examined and results of repA multiplex PCR.

\begin{tabular}{|c|c|c|c|c|}
\hline Bcc species/group & No. isolates & No. STs & 636 bp product & 237 bp product \\
\hline B. cepacia & 9 & 7 & + & - \\
\hline B. multivorans & 15 & 14 & - & - \\
\hline B. cenocepacia IIIA & 13 & 11 & + & + \\
\hline B. cenocepacia IIIB & 29 & 27 & + & + \\
\hline B. cenocepacia IIIC & 3 & 3 & - & - \\
\hline B. cenocepacia IIID & 2 & I & + & + \\
\hline B. stabilis & 5 & 3 & + & - \\
\hline B. vietnamiensis & 10 & 6 & + (I ST negative) & - \\
\hline B. dolosa & 3 & 3 & + & - \\
\hline B. ambifaria & 18 & 11 & + (I ST negative) & - \\
\hline B. anthina & 7 & 7 & + & - \\
\hline B. pyrrocinia & 5 & 5 & + (I ST negative) & - \\
\hline Bcc Group E & 3 & 3 & + & - \\
\hline Bcc Group K & 8 & 6 & + & - \\
\hline $\mathrm{Bcc} 2$ & 2 & I & + & - \\
\hline Bcc3 & 3 & 3 & + & - \\
\hline Bcc4 & 3 & 3 & + & - \\
\hline Bcc5 & 12 & 9 & - & - (3 STs positive) \\
\hline Bcc6 & 19 & 18 & + & - (7 STs positive) \\
\hline $\mathrm{Bcc} 8$ & 1 & 1 & + & - \\
\hline Total & 170 & 142 & & \\
\hline
\end{tabular}

The number of isolates and STs for each Bcc group or species is shown. Certain STs within these groups produced the opposite repA multiplex PCR result to that expected for either the 636 bp or 237 bp product; the number of STs and the PCR result for these non-conforming strains are indicated in brackets. 
cation and partitioning, respectively, of the multiple chromosomal replicons present in Bcc genomes [27].

Of these two genes, repA was the most suitable for the design of the identification method. The successful repA multiplex PCR developed was capable of identifying $B$. cenocepacia strains IIIA, IIIB and IIID; also its testing represents the first time a single identification test had been compared to a reference collection of Bcc strains identified and typed by MLST. The repA multiplex PCR method was primarily designed to detect the two main $B$. cenocepacia recA groups IIIA and IIIB, however, its efficacy for the identification of B. cenocepacia IIID should prove clinically useful. As opposed to B. cenocepacia IIIC which is entirely comprised of the environmental isolates which are specialist plant endophytes [20], recA groups IIIA, IIIB and IIID all include clinical isolates of $B$. cenocepacia [10$12,19,28$ ] and therefore are the most likely to be encountered in clinical microbiology practice.

The PCR method was tested on 142 different ST types. Its sensitivity reached $100 \%$ within the target groups of $B$. cenocepacia IIIA, IIIB and IIID, while specificity was found at $93 \%$ due to several strains from the Bcc6 producing the same PCR result as B. cenocepacia. Eight different Bcc groups of unassigned species status (Bcc Groups K, E, Bcc2 to 6 , and Bcc group 8 ) were included in the study making the strain collection examined one of the most broad in terms of Bcc diversity. While the Group K has already been shown to potentially comprise more than one bacterial unit analogous to the species level [16], the taxonomic positions of other Bcc groups remain unanswered. However, a recent study describing the Bcc groups 5 and 6 for the first time [15], showed that Bcc6 was very closely related to $B$. cenocepacia recA groups IIIA and IIIB suggesting their same species origin. Our PCR was able to detect 7 out of the 18 STs examined within Bcc6 (Table 2). Although this detection rate was below $50 \%$ it may prove useful in the future if Bcc6 is assigned to B. cenocepacia. In contrast we were able to eliminate the positivity of Bcc5 during the design of the repA-based PCR. Although Bcc5 has been proposed to be closely related to $B$. cenocepacia (like Bcc6, [15]), with repA analysis two Bcc5 isolates clustered most closely to B. cenocepacia IIIC and IIID, and a third isolate formed a distinct arm (Figure 2, panel A). Phylogenetic analysis of the concatenated sequences from seven MLST loci also show even closer links to $B$. stabilis and B. pyrrocinia rather than B. cenocepacia IIIA or IIIB [15] suggesting that resolution of the taxonomic status of Bcc5 will require further analysis.

The detection limit experiment demonstrated that the repA PCR was able to detect approximately $10^{6} \mathrm{CFU} / \mathrm{ml} B$. cenocepacia from a pure culture after 25 cycles of PCR. A similar detection limit has been observed with the stand- ard recA gene PCR for B. cepacia complex bacteria [22]. Once chronically infected, large numbers of $B$. cepacia complex bacteria may be present in CF sputum (ranging from $10^{5}$ to $10^{8} \mathrm{CFU} / \mathrm{ml}$; PD and EM unpublished data). The sensitivity of the repA PCR seen in this study indicates that without modification, it could only be applied directly to sputum for the most heavily infected individuals. However, our previous studies have shown that by nesting a recA gene-based PCR as low as $10^{3} \mathrm{CFU} / \mathrm{ml}$ of Bcc bacteria may be directly detected from CF sputum and identify individuals that are culture negative, but show early evidence of being infected [25]. Since we have now shown that repA is an accurate $B$. cenocepacia identification target, the design of future nested PCRs based on this gene may be undertaken. Overall, the repA PCR as developed herein is most suited to application on pure Bcc cultures and the specific identification of B. cenocepacia IIIA, IIIB and IIID.

Sequence analysis of repA as well as of another partitioning gene parB revealed that a degree of their sequence conservation was comparable with other house-keeping genes used for MLST. parB was more polymorphic than repA as the number of identified alleles within it was the second largest identified after MLST locus gyrB. Lower variation of the B. cenocepacia repA was observed in phylogenetic analysis where, unlike parB, B. cenocepacia IIIB formed a uniform single cluster which was adjacent to $B$. cenocepacia IIIA (Figure 2, panel A). Overall the repA tree closely mirrored the phylogenetic relatedness of the Bcc members observed by concatenated nucleotide sequences from seven MLST loci [14]. Phylogenetic trees of parB (Figure 2, panel B) or recA [13] do not clearly identify this close B. cenocepacia IIIA-IIIB relatedness.

Our analysis has also enabled us to speculate on the evolution of the multireplicon B. cepacia complex genome. An intriguing question of Bcc genomic biology is: has chromosomal replicon movement occurred between species? While considerable genomic plasticity mediated by insertion sequences and the capacity of strains to survive deletion of over $800 \mathrm{~kb}$ of DNA from secondary replicons has been shown for Bcc bacteria [29], we do not know if they are capable of swapping replicons between species. Examination of the full length $r e p A$ and $p a r B$ genes from complete Bcc genomes demonstrated clustering by chromosomal replicon, while each species possessed genetically distinct replication regions within these major clusters (Figure 1). This suggests that the second and third chromosomes present in Bcc each have a distinct common ancestor that was present in the Bcc progenitor prior to its divergence into the closely related species that currently make up the complex. Although we found evidence of different strains of a species (eg. multiple STs) sharing a given repA or parB allele, we did not find any that were 
shared by different Bcc species. These data suggest that the second chromosomal replicon has not moved between Bcc species and was evolutionary stabilised a long time ago such that it diverged in concert with their emergence as new species. It also indicates that the partitioning and replication regions of Bcc bacteria are highly stable and essential, such that inter-species recombination which has been observed for several Bcc MLST loci [30] does not occur in these regions.

\section{Conclusion}

In conclusion, we have developed easy-to-use single PCR method that is capable of unifying the detection of all $B$. cenocepacia strains except for recA subgroup IIIC. The repA gene targeted by this assay possesses sequence polymorphisms that enable Bcc species clustering which closely mirrors that provided by MLST and making repA a stable and discriminatory gene for the development of diagnostics of Bcc infection. We have also shown the second and third chromosomal replicons possessed by these bacteria have evolved stably with each respective Bcc species and that replicon movement has not occurred after their speciation.

\section{Methods \\ Bacterial strains}

Bcc isolates used in this study were derived from the Cardiff University collection and were subjected to growth, DNA extraction and basic identification as previously described [13]. In addition, the collection comprised highly divergent isolates representing all nine formally described Bcc species as well as novel Bcc groups identified by MLST: Bcc Group K $[16,21]$, Bcc groups 2 - 6 $[14,15]$ and Bcc group 8 (A. Baldwin, unpublished data). In total, 215 isolates were examined at the repA locus; 83 isolates (corresponding to 67 MLST sequence type; STs) were subjected to repA sequence analysis; for testing the repA-based PCR this was expanded to 170 isolates (142 STs) including 38 of those that were sequenced. 120 isolates (95 STs) from the same collection were sequenced for parB analysis.

Ten non-Bcc bacteria reference isolates representative of closely related species or those that are frequently encountered in CF respiratory samples were used as controls for the molecular diagnostics: Achromobacter xylosoxidans LMG 1863' ${ }^{\mathrm{T}}$ Burkholderia gladioli LMG 2216 ${ }^{\mathrm{T}}$, Delftia acidovorans LMG 1226 ${ }^{\mathrm{T}}$, Pseudomonas aeruginosa PAO1, Pseudomonas fluorescens LMG $1794^{\mathrm{T}}$, Pseudomonas putida LMG 2257 ${ }^{\mathrm{T}}$, Pseudomonas stutzeri LMG $11199^{\mathrm{T}}$, Ralstonia pickettii LMG 5942 ${ }^{\mathrm{T}}$, Ralstonia mannitolytica LMG $6866^{\mathrm{T}}$ and Stenotrophomonas maltophilia LMG 958T; LMG strains were obtained from Belgium Coordinated Collections of Microorganisms [31].

\section{Amplification and sequence analysis of repA and parB}

The repA and parB sequences [27] from the second and third chromosomes, and plasmid of B. cenocepacia strain J2315 were downloaded from the genome sequence of this strain produced by the Pathogen Sequencing Group at the Sanger Institute, Hinxton, Cambridge [32]. The Basic Local Alignment Search Tool (BLAST; [33]) was then used to identify homologous genes in the genomes of five additional Bcc strains produced by the US Department of Energy Joint Genome Institute [34]: B. cenocepacia IIIB strains AU1054 and HI2424, Bcc Group K strain 383, B. vietnamiensis G4 and B. ambifaria AMMD. Annotated repA and parB genes from the published genomes of Burkholderia pseudomallei [35], Burkholderia mallei [36] and Burkholderia xenovorans [37] were included in selected analyses as reference sequences. Alignment and phylogenetic analysis of DNA sequences was performed using the BioEdit [38] and Mega 3.1 [39] softwares as described previously [40]. Phylogenetic trees were primarily drawn using Neighborjoining method (Jukes-Cantor algorithm) and bootstrap analysis evaluated for 500 replicates. Three additional treeing algorithms were evaluated (UPGMA, Minimum Evolution and Maximum Parsimony) and produced phylogenetic trees that were concordant with final Neighborjoining methods presented in the study. In addition to the DNA-based analysis, translated amino acid sequences were also used for the tree reconstruction, however no discrepancy with the nucleotide-based trees was found.

Universal Bcc primers for amplification of the repA gene encoded on the second chromosome were designed from the alignments (primers repA-UNI-5 and repA-UNI-3; Table 1). The universal primers were used to amplify the 898 bp region of the Bcc repA gene from 83 isolates representing 67 divergent STs. PCR was performed in a 20- $\mu \mathrm{l}$ reaction mixture containing $2.0 \mathrm{mM} \mathrm{MgCl} 2,0.2 \mathrm{mM}$ of each dNTPs, $0.8 \mathrm{U}$ of Taq polymerase (Promega, Madison, USA), $0.5 \mu \mathrm{M}$ of each primers (Table 1 ) and $1 \mu \mathrm{l}$ of template DNA (approximately $500 \mathrm{ng}$ ). The PCR was performed on a Dyad DNA Engine thermal cycler (Bio-Rad Laboratories, Hemel Hempstead, UK). The thermal cycling profile was repeated 35 times and consisted of 30 sec at $94^{\circ} \mathrm{C}, 45 \mathrm{sec}$ at $55^{\circ} \mathrm{C}$ and $1 \mathrm{~min}$ at $72^{\circ} \mathrm{C}$. The amplified repA-gene products were sequenced in both directions using internal sequencing primers (repA-SEQ- 5 and repASEQ-3; Table 1) exactly as described [14]. The maximal available accurate repA sequence read was used for species-specific primer design (see below), however for phylogenetic analysis a subset of 73 sequences were trimmed to a single overlapping region of $345 \mathrm{bp}$.

In an analogous fashion to the repA gene analysis described above, universal primers were designed to amplify the parB gene encoded on the Bcc second chromosome (parB-UNI-5 and parB-UNI-3; Table 1). A 656 bp 
parB amplification product was produced using PCR conditions identical to those for repA. Sequence analysis of 120 isolates (95 STs) was performed on both strands using the universal parB primers (Table 1) as described. Phylogenetic analysis was performed on a subset of 114 sequences trimmed to an overlapping internal region of 494 bp.

\section{B. cenocepacia species-specific PCR targeting repA gene} A multiplex PCR producing two repA gene fragments of 636 and 237 bp in length was designed to distinguish $B$. cenocepacia from all other Bcc members. The multiplex PCR used the repA sequencing 5' primer (repA-SEQ-5; Table 1) and two different 3' primers (repA-636-3 and repA-237-3, Table 1 ). PCR was carried out in a $20-\mu$ l reaction volume containing $1.5 \mathrm{mM} \mathrm{MgCl}_{2}, 0.2 \mathrm{mM}$ (each) dNTPs, $0.75 \mathrm{U}$ of Taq polymerase (Promega, Madison, USA), $0.72 \mu \mathrm{M} 5$ ' primer, $0.5 \mu \mathrm{M}$ 3' primer repA-636-3, $0.22 \mu \mathrm{M} 3$ ' primer repA-237-3 and $0.5 \mu \mathrm{l}$ of template DNA (approximately $250 \mathrm{ng}$ ). The PCR program was run on a Dyad DNA Engine thermal cycler (Bio-Rad Laboratories, Hemel Hempstead, UK) with initial denaturation 5 min at $94^{\circ} \mathrm{C}$ and a subsequent run of 20 cycles, each comprising $30 \mathrm{sec}$ at $94^{\circ} \mathrm{C}, 15 \mathrm{sec}$ at annealing temperature and $15 \mathrm{sec}$ at $72^{\circ} \mathrm{C}$. The initial annealing temperature set at $70^{\circ} \mathrm{C}$ was decreasing for the first 10 cycles by $0.2^{\circ} \mathrm{C} /$ cycle to reach the final annealing temperature of $68^{\circ} \mathrm{C}$. The PCR specificity and sensitivity was evaluated by testing $170 \mathrm{Bcc}$ isolates falling into 142 different STs (Table 2); in addition the 10 non-Bcc species commonly found in sputum were also tested (see above).

The detection limit of the assay was determined by serial decimal dilutions of DNA extracted from a B. cenocepacia culture of known viability characterized in CFU/mL. The reference strain B. cenocepacia J2315 was grown in Luria Bertani broth to an optical density $(630 \mathrm{~nm})$ of 0.5 units, corresponding to a viability of approximately $5 \times 10^{8}$ $\mathrm{CFU} / \mathrm{ml}$. Serial dilutions of this culture were plated as 10 $\mu$ l drop counts onto Tryptic Soya Agar and incubated overnight at $37^{\circ} \mathrm{C}$ to precisely determine the number of viable cells present. In parallel, $200 \mu \mathrm{l}$ of each dilution were mixed with $100 \mu \mathrm{l}$ of 5\% Chelex 100 and a rapid DNA extraction performed exactly as described [40]; $1 \mu \mathrm{l}$ of this DNA extraction was then subjected to either 20 or 25 cycles of PCR as described above. Once viable counts were available, all the dilutions performed in making the DNA extraction and setting up the PCR were taken into account to calculate the number of viable cells present in the detection limit experiment.

\section{Nucleotide accession numbers}

Representative repA and parB allele sequences determined in this study were deposited in GenBank under accession numbers EU165089 to EU165123 and EU165124 to EU165190, respectively.

\section{Authors' contributions}

PD performed the PCR-based experiments and testing of repA method. $\mathrm{AB}$ and $\mathrm{PD}$ performed the sequence analyses. EM, PD, AB and CGD drafted the manuscript. All authors conceived of the study, participated in its design, read and approved the final manuscript.

\section{Additional material}

\section{Additional file 1}

Sequences repA. Sequences of the repA gene retrieved for 83 Burkholderia cepacia complex isolates (67 different STs) are provided in multifasta format after performing the multiple alignment. Each sequence title contains the internal number, genomovar and ST number of the organism it came from. Reference sequences from 9 Burkholderia strains with finished genomes are also included.

Click here for file

[http://www.biomedcentral.com/content/supplementary/14712180-8-44-S1.txt]

\section{Additional file 2}

Sequences parB. Sequences of the parB gene retrieved for 120 Burkholderia cepacia complex isolates (95 different STs) are provided in multifasta format after performing the multiple alignment. Each sequence title contains the internal number, genomovar and ST number of the organism it came from. Reference sequences from 9 Burkholderia strains with finished genomes are also included.

Click here for file

[http://www.biomedcentral.com/content/supplementary/1471-

2180-8-44-S2.txt]

\section{Acknowledgements}

We thank Angela Marchbank for excellent technical assistance. This work was funded by grants from the Wellcome Trust (grant 075586 E.M.; grant 072853; C.G.D. and E.M.) and UK Cystic Fibrosis Trust (grant PJ535).

\section{References}

I. LiPuma JJ: Burkholderia cepacia. Management issues and new insights. Clin Chest Med 1998, 19(3):473-86, vi.

2. Mahenthiralingam E, Urban TA, Goldberg JB: The multifarious, multireplicon Burkholderia cepacia complex. Nat Rev Microbiol 2005, 3(2): 144-156.

3. Vandamme P, Holmes B, Vancanneyt M, Coenye T, Hoste B, Coopman R, Revets H, Lauwers S, Gillis M, Kersters K, Govan JR: Occurrence of multiple genomovars of Burkholderia cepacia in cystic fibrosis patients and proposal of Burkholderia multivorans sp. nov. Int J Syst Bacteriol I997, 47(4): I I 88- I 200.

4. Vandamme P, Holmes B, Coenye T, Goris J, Mahenthiralingam E, LiPuma JJ, Govan JR: Burkholderia cenocepacia sp. nov.--a new twist to an old story. Res Microbiol 2003, I54(2):91-96.

5. Vandamme P, Mahenthiralingam E, Holmes B, Coenye T, Hoste B, De Vos $P$, Henry D, Speert DP: Identification and population structure of Burkholderia stabilis sp. nov. (formerly Burkholderia cepacia genomovar IV). J Clin Microbiol 2000, 38(3): I042- 047.

6. Vermis K, Coenye T, LiPuma JJ, Mahenthiralingam E, Nelis HJ, Vandamme P: Proposal to accommodate Burkholderia cepacia genomovar VI as Burkholderia dolosa sp. nov. Int J Syst Evol Microbiol 2004, 54(Pt 3):689-69I. 
7. Coenye T, Mahenthiralingam E, Henry D, LiPuma J], Laevens S, Gillis M, Speert DP, Vandamme P: Burkholderia ambifaria sp. nov., a novel member of the Burkholderia cepacia complex including biocontrol and cystic fibrosis-related isolates. Int J Syst Evol Microbiol 200I, 5 I (Pt 4): I 48I-I 490.

8. Vandamme P, Henry D, Coenye T, Nzula S, Vancanneyt M, LiPuma J], Speert DP, Govan JR, Mahenthiralingam E: Burkholderia anthina sp. nov. and Burkholderia pyrrocinia, two additional Burkholderia cepacia complex bacteria, may confound results of new molecular diagnostic tools. FEMS Immunol Med Microbiol 2002, 33(2): 143-149.

9. Brisse S, Cordevant C, Vandamme P, Bidet P, Loukil C, Chabanon G, Lange $M$, Bingen E: Species distribution and ribotype diversity of Burkholderia cepacia complex isolates from French patients with cystic fibrosis. I Clin Microbiol 2004, 42(10):4824-4827.

10. LiPuma JJ, Spilker T, Gill LH, Campbell PW 3rd, Liu L, Mahenthiralingam E: Disproportionate distribution of Burkholderia cepacia complex species and transmissibility markers in cystic fibrosis. Am J Respir Crit Care Med 200I, I 64(I):92-96.

II. Speert DP, Henry D, Vandamme P, Corey M, Mahenthiralingam E: Epidemiology of Burkholderia cepacia Complex in Patients with Cystic Fibrosis, Canada. Emerg Infect Dis 2002, 8(2): | $18 \mid-187$.

12. Reik R, Spilker T, Lipuma J]: Distribution of Burkholderia cepacia complex species among isolates recovered from persons with or without cystic fibrosis. J Clin Microbiol 2005, 43(6):2926-2928.

13. Mahenthiralingam E, Bischof J, Byrne SK, Radomski C, Davies JE, AvGay Y, Vandamme P: DNA-Based diagnostic approaches for identification of Burkholderia cepacia complex, Burkholderia vietnamiensis, Burkholderia multivorans, Burkholderia stabilis, and Burkholderia cepacia genomovars I and III. J Clin Microbiol 2000, 38(9):3165-3173.

14. Baldwin A, Mahenthiralingam E, Thickett KM, Honeybourne D, Maiden MC, Govan JR, Speert DP, Lipuma J], Vandamme P, Dowson CG: Multilocus sequence typing scheme that provides both species and strain differentiation for the Burkholderia cepacia complex. J Clin Microbiol 2005, 43(9):4665-4673.

15. Dalmastri C, Baldwin A, Tabacchioni S, Bevivino A, Mahenthiralingam E, Chiarini L, Dowson C: Investigating Burkholderia cepacia complex populations recovered from Italian maize rhizosphere by multilocus sequence typing. Environ Microbiol 2007 , 9(7):1632-1639.

16. Mahenthiralingam E, Baldwin A, Drevinek $P$, Vanlaere E, Vandamme $P$, Lipuma JJ, Dowson CG: Multilocus sequence typing breathes life into a microbial metagenome. PLoS ONE 2006, I:e I7.

17. Drevinek P, Vosahlikova S, Cinek O, Vavrova V, Bartosova J, Pohunek $P$, Mahenthiralingam E: Widespread clone of Burkholderia cenocepacia in cystic fibrosis patients in the Czech Republic. Med Microbiol 2005, 54(Pt 7):655-659.

18. Mahenthiralingam E, Vandamme $P$, Campbell ME, Henry DA, Gravelle AM, Wong LT, Davidson AG, Wilcox PG, Nakielna B, Speert DP: Infection with Burkholderia cepacia complex genomovars in patients with cystic fibrosis: virulent transmissible strains of genomovar III can replace Burkholderia multivorans. Clin Infect Dis 200I, 33(9): |469-1475.

19. Manno G, Dalmastri C, Tabacchioni S, Vandamme P, Lorini R, Minicucci L, Romano L, Giannattasio A, Chiarini L, Bevivino A: Epidemiology and clinical course of Burkholderia cepacia complex infections, particularly those caused by different Burkholderia cenocepacia strains, among patients attending an Italian Cystic Fibrosis Center. J Clin Microbiol 2004, 42(4): I49|-I 497.

20. Balandreau J, Viallard V, Cournoyer B, Coenye T, Laevens S, Vandamme P: Burkholderia cepacia genomovar III Is a common plant-associated bacterium. Appl Environ Microbiol 2001, 67(2):982-985.

21. Vermis K, Coenye T, Mahenthiralingam E, Nelis HJ, Vandamme P: Evaluation of species-specific recA-based PCR tests for genomovar level identification within the Burkholderia cepacia complex. J Med Microbiol 2002, 5 I (I I):937-940.

22. LiPuma JJ, Dulaney BJ, McMenamin JD, Whitby PW, Stull TL, Coenye T, Vandamme P: Development of rRNA-based PCR assays for identification of Burkholderia cepacia complex isolates recovered from cystic fibrosis patients. I Clin Microbiol 1999, 37(10):3167-3170.
23. McDowell A, Mahenthiralingam E, Moore JE, Dunbar KE, Webb AK, Dodd ME, Martin SL, Millar BC, Scott CJ, Crowe M, Elborn JS: PCRBased Detection and Identification of Burkholderia cepacia Complex Pathogens in Sputum from Cystic Fibrosis Patients. J Clin Microbiol 200I, 39( I 2):4247-4255.

24. McMenamin JD, Zaccone TM, Coenye T, Vandamme P, LiPuma J]: Misidentification of Burkholderia cepacia in US cystic fibrosis treatment centers: an analysis of $I, 05 I$ recent sputum isolates. Chest 2000, II7(6):1661-1665.

25. Drevinek P, Hrbackova H, Cinek O, Bartosova J, Nyc O, Nemec A, Pohunek P: Direct PCR Detection of Burkholderia cepacia Complex and Identification of Its Genomovars by Using Sputum as Source of DNA. J Clin Microbiol 2002, 40(9):3485-3488.

26. Baldwin A, Mahenthiralingam E, Drevinek $P$, Vandamme $P$, Govan JRW, Waine DJ, LiPuma JJ, Chiarini L, Dalmastri C, Henry D, Speert DP, Honeybourne D, Maiden CJ, Dowson CG: Environmental Burkholderia cepacia complex isolates in human infection. Emerg Infect Dis 2007, 13(3):458-46I.

27. Dubarry N, Pasta F, Lane D: ParABS systems of the four replicons of Burkholderia cenocepacia: new chromosome centromeres confer partition specificity. J Bacteriol 2006, | 88(4): | 489 - I 496.

28. Agodi A, Mahenthiralingam E, Barchitta M, Giannino V, Sciacca A, Stefani S: Burkholderia cepacia complex infection in Italian patients with cystic fibrosis: prevalence, epidemiology, and genomovar status. J Clin Microbiol 200I, 39(8):289 I-2896.

29. Lessie TG, Hendrickson W, Manning BD, Devereux R: Genomic complexity and plasticity of Burkholderia cepacia. FEMS Microbiol Lett 1996, 144(2-3): I I7-128.

30. Waine DJ, Henry DA, Baldwin A, Speert DP, Honeybourne D, Mahenthiralingam E, Dowson CG: Reliability of multilocus sequence typing of the Burkholderia cepacia complex in cystic fibrosis. J Cyst Fibros 2007, 6(3):215-219.

3I. Belgian Co-ordinated Collections of Micro-organisms [http:/ lbccm.belspo.be/]

32. Sanger Institute - Burkholderia cenocepacia [http:// www.sanger.ac.uk/Projects/B cenocepacia/]

33. Altschul SF, Madden TL, Schaffer AA, Zhang J, Zhang Z, Miller W, Lipman DJ: Gapped BLAST and PSI-BLAST: a new generation of protein database search programs. Nucleic Acids Res 1997, 25(I7):3389-3402.

34. DOE Joint Genome Institute [http://www.jgi.doe.gov/]

35. Holden MT, Titball RW, Peacock S], Cerdeno-Tarraga AM, Atkins T, Crossman LC, Pitt T, Churcher C, Mungall K, Bentley SD, Sebaihia M, Thomson NR, Bason N, Beacham IR, Brooks K, Brown KA, Brown NF, Challis GL, Cherevach I, Chillingworth T, Cronin A, Crossett B, Davis P, DeShazer D, Feltwell T, Fraser A, Hance Z, Hauser H, Holroyd S, Jagels K, Keith KE, Maddison M, Moule S, Price C, Quail MA, Rabbinowitsch E, Rutherford K, Sanders M, Simmonds M, Songsivilai $S$, Stevens K, Tumapa S, Vesaratchavest M, Whitehead S, Yeats C, Barrell BG, Oyston PC, Parkhill J: Genomic plasticity of the causative agent of melioidosis, Burkholderia pseudomallei. Proc Natl Acad Sci U S A 2004, I0 I(39): I 4240- I 4245.

36. Nierman WC, DeShazer D, Kim HS, Tettelin H, Nelson KE, Feldblyum T, Ulrich RL, Ronning CM, Brinkac LM, Daugherty SC, Davidsen TD, Deboy RT, Dimitrov G, Dodson RJ, Durkin AS, Gwinn ML, Haft DH, Khouri H, Kolonay JF, Madupu R, Mohammoud Y, Nelson WC, Radune D, Romero CM, Sarria S, Selengut J, Shamblin C, Sullivan SA, White O, Yu Y, Zafar N, Zhou L, Fraser CM: Structural flexibility in the Burkholderia mallei genome. Proc Natl Acad Sci U S A 2004, I0I(39): |4246-|425|.

37. Chain PS, Denef VJ, Konstantinidis KT, Vergez LM, Agullo L, Reyes VL, Hauser L, Cordova M, Gomez L, Gonzalez M, Land M, Lao V, Larimer F, LiPuma J], Mahenthiralingam E, Malfatti SA, Marx C], Parnell J], Ramette A, Richardson P, Seeger M, Smith D, Spilker T, Sul WJ, Tsoi TV, Ulrich LE, Zhulin IB, Tiedje JM: Burkholderia xenovorans LB400 harbors a multi-replicon, 9.73-Mbp genome shaped for versatility. Proc Natl Acad Sci U S A 2006, I 03(42): I 5280-I 5287.

38. BioEdit Sequence Alignment Editor [http:// www.mbio.ncsu.edu/BioEdit/bioedit.html]

39. MEGA - Molecular Evolutionary Genetics Analysis [http:// www.megasoftware.net/]

40. Payne GW, Vandamme P, Morgan SH, LiPuma IJ, Coenye T, Weightman AJ, Jones TH, Mahenthiralingam E: Development of a recA Gene-Based Identification Approach for the Entire Burkholderia Genus. Appl Environ Microbiol 2005, 7 I (7):3917-3927. 